\title{
The Role Cultural Competency Plays in Teaching Data Science
}

\author{
Kelly Gaither \\ The University of Texas at Austin \\ kelly@tacc.utexas.edu
}

\author{
Lizanne DeStefano \\ Georgia Institute of Technology \\ ldestefano6@gatech.edu
}

\author{
Rosalia Gomez \\ The University of Texas at Austin \\ $\underline{\text { rosie@tacc.utexas.edu }}$
}

Lorna Rivera

Georgia Institute of Technology

lorna.rivera@ceismc.gatech.edu

\author{
Helen Turner \\ Chaminade University of Honolulu \\ hturner@chaminade.edu
}

\begin{abstract}
Supporting Pacific Indigenous Computing Excellence (SPICE) is based on unique expertise and proven models established through a partnership between the Texas Advanced Computing Center at the University of Texas at Austin, Chaminade University of Honolulu and Georgia Institute of Technology (Georgia Tech). The SPICE program leverages shared partnership experiences to address two goals: 1) Perform original research and program development to bridge computation and culture -- developing culturally-consistent conceptual and practical frameworks for thinking about big data problems and communicating student outcomes and attainment to family, community and kupuna (Hawaiian wisdom figures); and 2) Implement an in situ Data Science, Analytics and Visualization (DSAV) Summer Immersion Experience (SIE) as a summer program in Hawai' $i$ to provide a month-long summer immersion program in data science, visualization, and virtual reality to Native Hawaiian and Pacific Islander (NHPI) and disadvantaged students. In this paper, we present the framework for this effort, with relevant educational, and cultural research to justify decisions made to date.
\end{abstract}

\section{CCS CONCEPTS}

- Computing Education $\rightarrow$ Computational thinking; Computing education programs; Computer science education, Computational science and engineering education

\section{KEYWORDS}

Permission to make digital or hard copies of all or part of this work for personal or classroom use is granted without fee provided that copies are not made or distributed for profit or commercial advantage and that copies bear this notice and the full citation on the first page. Copyrights for components of this work owned by others than ACM must be honored. Abstracting with credit is permitted. To copy otherwise, or republish, to post on servers or to redistribute to lists, requires prior specific permission and/or a fee. Request permissions from permissions@acm.org.

PEARC '18, July 22-26, 2018, Pittsburgh, PA, USA

(C) 2018 Association for Computing Machinery.

ACM ISBN 978-1-4503-6446-1/18/07 ...\$15.00

https://doi.org/10.1145/3219104.3219160
L.0 Science and Technology of Learning, L.0 Assessment/Evaluation/Measurement, L1.2 Learning Objects, L.2 Learning.

\section{ACM Reference format:}

K. Gaither, R. Gomez, H. Turner, L. DeStefano, L. Rivera, and M. Bland. 2018. The Role Cultural Competency Plays in Teaching Data Science. In Proceedings of Practice and Experience in Advanced Research Computing (PEARC '18). ACM, New York, NY, USA, 7 pages. https://doi.org/10.1145/3219104.3219160

\section{INTRODUCTION}

Native Hawaiians and Pacific Islanders (NHPI) are the least represented group of those recognized by the United States (US) government agencies as underrepresented in the fields of Science, Technology, Engineering and Mathematics (STEM). A 2016 report from the National Science Foundation (NSF) workshop on "Recognizing and Removing Barriers to STEM careers for Native Hawaiians and Pacific Islanders," outlined the barriers to STEM careers for NHPI. Listed first was cultural barriers, including language, relevance, and connecting methodology to a sense of place [1]. As noted in the report, addressing this barrier is dependent on increasing indigenous participation in STEM while simultaneously developing curriculum and expertise that is culturally competent in the NHPI languages, traditions, and customs.

As defined by the National Education Association, "cultural competence is having an awareness for one's own cultural identity and views about difference, and the ability to learn and build on the varying cultural and community norms of students and their families."1 Research on culturally relevant pedagogy suggests that people from underrepresented communities have fewer opportunities to connect personally with STEM practices and activities they encounter in formal educational settings. Typical instructional practices do not afford students the opportunities to draw from their personal and cultural knowledge of science and

\footnotetext{
${ }^{1}$ http://www.nea.org/home/39783.htm
} 
math, contributing to alienation and a decreased likelihood of pursuing STEM-related fields [2].

Technology, however, offers the unique chance for educators to bridge the curriculum of school to the 21st century learner. Bridging the disconnect between school learning and the realworld needs of students, particularly underrepresented students, is the most significant barrier. Computers, when used well, "can provide students with an excellent tool for applying concepts in a variety of contexts, thereby breaking the artificial isolation of school subject matter from the real-world situations [3]." Conole et al. noted that today's student is continuously connected and for them, technology is transferable, integrated, personalized, organized, adaptive, and pervasive [4]. This suggests that infusing technology into STEM curriculum in a meaningful manner becomes truly relevant and responsive to the learner of the $21 \mathrm{st}$ century-meaning learning in school mirrors the learning students engage in outside of school [5].

In this paper, we detail our NSF funded INCLUDES design and development launch pilot project, SPICE, Supporting Pacific Indigenous Computing Excellence, to develop a culturally competent data science immersion experience and curriculum that connects students to their real-world lives. The project is a collaboration between Native Hawaiians, local Hawaiian educators and research leaders at Chaminade University of Honolulu, technology educators and researchers at the Texas Advanced Computing Center (TACC) and evaluators from Georgia Tech University. The SPICE vision is to train a cadre of Native Hawaiian (öiwi) and Pacific Islander (Pasifika) STEM students who are positioned to lead data science, visualization and analytics efforts that support health, sustainability and social justice in the Hawaiian Lāhui (homeland) and the US-affiliated Pacific.

\section{HAWAIIAN CULTURAL DISCONNECTS FROM STEM}

There are a number of prevalent reasons why öiwi-Pasifika students have been historically disenfranchised from STEM. Perhaps best understood are socioeconomic and academic preparation issues associated with high poverty levels and systemic trauma relating to loss of aina (land) and selfdetermination [6, 7]. These issues have been the focus of numerous programs to increase STEM participation and are of high importance. However, they are far from the whole story. Next level issues, which are harder to address, derive from the high level of colonization of the science endeavor with principles and operating practices that are antithetical to the Hawaiian worldview and the sociocultural norms of Pacific Islanders. Higher education is colonized and does not support indigeneity, with a dearth of role models and cultural inclusivity. Lastly, the STEM endeavor has a tortured relationship with the Native Hawaiian community, with conflicts between science and culture illustrated by clashes over geothermal exploration, GMO foods and the Thirty Meter Telescope [8]. Young Hawaiians, especially if educated at Hawaiian-focused or immersion schools, have selfefficacy as representatives of a culture that managed resources 2 using ahupua'a, practiced aquaculture, engineered voyaging canoes and navigated the oceans. However, as they encounter higher STEM education, the idea of a Hawaiian scientist is redefined, usually as a necessary adoption of a non-indigenous identity [9-11]. Not unlike the 'unnecessary polarization' of faith and science that is alluded to by Alexander [12], this leads to the perception that participation in Western science is fundamentally 'un-Hawaiian' and that the practice of science is not culturally consistent. This translates to a potential lost opportunity to train and empower NHPI STEM professionals to enrich the Pacific science endeavor through engaging in an intensely regional science (place-based, culturally enmeshed, community-driven) while contributing to addressing global challenges for which the Pacific region is a sentinel site. The consequence of this perception is that oiwi-Pasifika who choose a STEM career experience 'outsider' status in community and family (ohana) and feel that they are forced to choose a new ohana. The primacy of family and community in NHPI culture cannot be underestimated, and therefore the consequences of alienation are significant $[10$, $13,14]$.

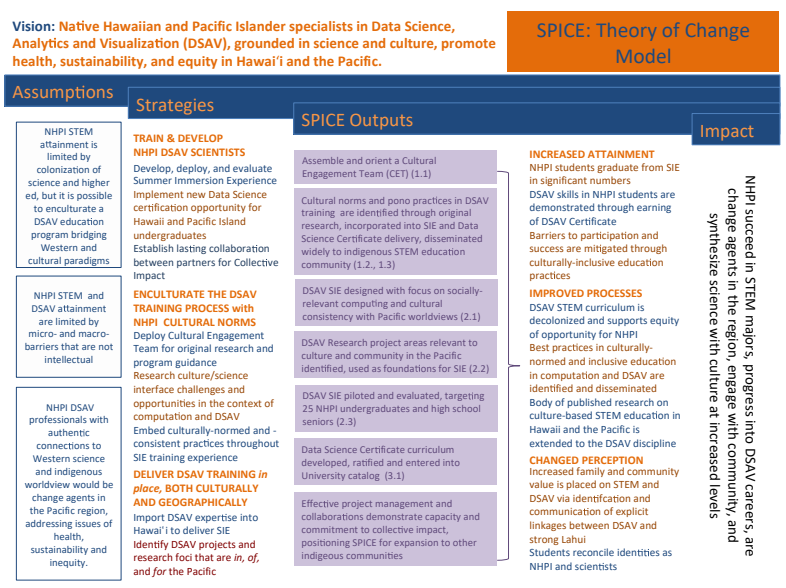

Figure 1: SPICE Theory of Change Model

This work builds on multiple programs at Chaminade University of Honolulu (and other educational organizations in Hawai'i) that incorporate culture and place-based learning in STEM curricula, primarily via provision of research opportunities and curriculum where relevance is established-focusing on questions and topics that can be viewed through a Hawai' $i$-Pacific lens in terms of either local relevance or cultural context. Examples of these approaches are the incorporation of la'au lapa'au traditional medicine in Chaminade's upper division pharmacology course, the teaching of ahupuaa land use models in an ecology course and the use of loko i'a fishpond or lo' $i$ taro patches to examine biodiversity in a core biology course.

We chose to focus on the computational disciplines that, a priori, seem particularly distant from traditional practices and current community needs. The SPICE Theory of Change in Figure 1 presents an approach that includes öiwi-Pasifika enculturation of Data Science, Analytics and Visualization (DSAV) training activities through both original research, and 
implementation of innovative activities designed by our cultural engagement team. We assert that NHPI underrepresentation in STEM fields, and in particular in cutting-edge data science, limits self-determination, economic development and social justice goals for Pacific indigenous communities. NHPI communities are affected by poverty, health disparities, environmental degradation and erosion of traditional culture $[10,15,16]$. NHPI are broadly disenfranchised from STEM, with STEM participation for these communities undermined by socioeconomic factors and a complex set of cultural and family factors that position STEM careers as outside, or at odds, with indigenous identity, and higher education as a source of alienation from family, community and culture. Moreover, without intervention, even larger nextgeneration STEM educational disparities loom for Pacific peoples.

\section{SPICE FRAME-WORK}

The SPICE vision is to train a cadre of Native Hawaiian (oiwi) and Pacific Islander (Pasifika) STEM students who are positioned to lead data science, visualization and analytics efforts that support health, sustainability and social justice in the Hawaiian Lăhui (homeland) and the US-affiliated Pacific. We recognize that agency over, and command of, big data is central to the Native Hawaiian and Pacific Islander (NHPI) self-determination.

We have developed the SPICE framework to be culturally responsive with a pedagogy that recognizes the importance of including student's cultural references in all aspects of teaching. Following the model set forth by Ladson-Billings, we have developed SPICE teaching to include [17]:

1. Positive perspectives on parents and families parents and families are the student's teacher and are critically important to their long-term success.

2. Communication of high expectations - all learners should be held to high expectations and given the appropriate resources to achieve at a rigorous standard.

3. Learning within the context of culture culture is a vital part of the human experience and influences every aspect of our lives, including learning.

4. Student-centered instruction - engaging the student's interests and passions facilitates a more engaged learning experience and allows students to learn skills in the context of their chosen interests.

5. Culturally mediated instruction - incorporating the diverse ways of knowing, understanding and representing information.

6. Reshaping the curriculum - as a means of integrating culture and cultural understanding into the curriculum.

7. Teacher as facilitator - understanding student culture, student interests and facilitating them in the learning process rather than presenting learning material and expecting students to determine how they relate.
Working in the Pacific indigenous context, we have developed a cultural framework to contextualize the SPICE through a host culture lens. 'Ōlelo No'eau (Native Hawaiian wisdom sayings) [18] that frame the SPICE program are as follows: 'A'ohe hua o ka mai'a i ka lā ho'okahi: no task is too big when done together by all. Team Science and collaborative approaches are central to data science and are embedded in the SPICE pedagogy. Hōhonu no ke kawa: a deep diving place, a topic that requires deep thinking. Data science, visualization and analytics allow students to approach intellectual and societal challenges at new levels of depth. SPICE leverages the power of these approaches to the benefit of the Lăhui_(Hawaiian homeland) and develops empowered NHPI to find data-driven solutions to problems of the Pacific.

Big Data and the Digital Divide. Since the molecular biology revolution of the $80 \mathrm{~s}$ and $90 \mathrm{~s}$, the pace of scientific change has accelerated, largely powered by the synergy of computational power and the 'big data' approach that embraces complexity and pattern-finding rather than painstaking reductionism. In all areas of society, the digital divide is troubling, but for STEM education, it creates a new challenge. For marginalized groups, such as NHPI, that only recently gained basic access to science pipelines, the goalposts have now shifted on what an effective preparation looks like with data literacy being critical. Unfortunately, the primarily undergraduate and community college institutions that serve these populations are, almost by definition, those least likely to offer data science curricula backed by high performance computing and expert faculty with a computational biology research agenda. Thus a 'big data, small school' challenge is emerging in minority STEM education. We believe that this paradox can only be addressed by new forms of inter-institutional partnership that melds an understanding of diversity and indigenous issues found in the small, undergraduate institution situated locally and a deep understanding of data science and analytic/visualization with partners across the nation.

Socioeconomic Disparities in the Pacific. Native Hawaiians (NH) are underrepresented in higher education and professional careers, and the NH community is affected by poverty, health disparities, domestic violence and incarceration [10, 19, 20]. Over $20 \%$ of $\mathrm{NH}$ live in poverty and $16 \%$ lack health coverage. Hawaiians suffer disproportionately from health burdens such as obesity, cancer, diabetes and cardiovascular disease ( $\mathrm{NH}$ obesity rates are $>40 \%$ in $25-40$ year old adults), and they smoke, contract tuberculosis, drop out of school and are incarcerated at some of the highest rates of any ethnicity in the nation [10]. Hawaiians are initially disenfranchised by a troubled public-school system: Hawai' i public schools have the shortest school year in the nation, rank $49^{\text {th }}$ in performance and NH student test scores in sciences are exemplified by less than $12 \%$ proficiency at the $9^{\text {th }}$ grade level [10]. These factors translate to underrepresentation and poor persistence in higher education, therefore, the Hawaiian community struggles to find the leadership and academic voice needed to confront its inequities. There are less than 10,000 NH in total with graduate degrees of any kind, $\sim 500 \mathrm{NH}$ with a $\mathrm{PhD}$, and less than $100 \mathrm{NH}$ with a science $\mathrm{PhD}[15,16,20,21]$. There are 103 Hawaiian physicians serving a Hawaiian population of 
$>0.5 \mathrm{M}$. NHPI. Together with Alaskan Natives and American Indians, they account for only $2.5 \%$ of US STEM majors (NSF).

Challenges faced by Hawaiians in Hawai' $i$ are exacerbated for indigenous islanders in the US-affiliated Pacific by dependence on economic aid, economies that cannot sustain educational, health or environmental services, geographical isolation and population dispersion. For example, Chuuk (Federated States of Micronesia)has a population of 43,000 across 208 islands and only three high schools. Forty percent (40\%) of children do not complete primary school, 20\% do not complete high school, and less than 3\% have four-year degrees. Roughly $35 \%$ of adult Chuukese have less than a high school education [1], and there are $\sim 4$ Chuukese with $\mathrm{PhDs}$ with 2 in environmental science $\mathrm{PhD}$ programs. The per capita GDP in Chuuk is USD $\$ 1,600$ p.a. and there is $85 \%$ unemployment. Such nations have stunning public health challenges (e.g. in some Pacific islands $>75 \%$ adults are obese, infant and maternal mortality are both 5 times US rates) [1, 22]. Pacific islands face health disparities, economic dependence on aid and climate uncertainty [23, 24], without widespread empowerment from higher education or a cadre of Pacific STEM professionals.

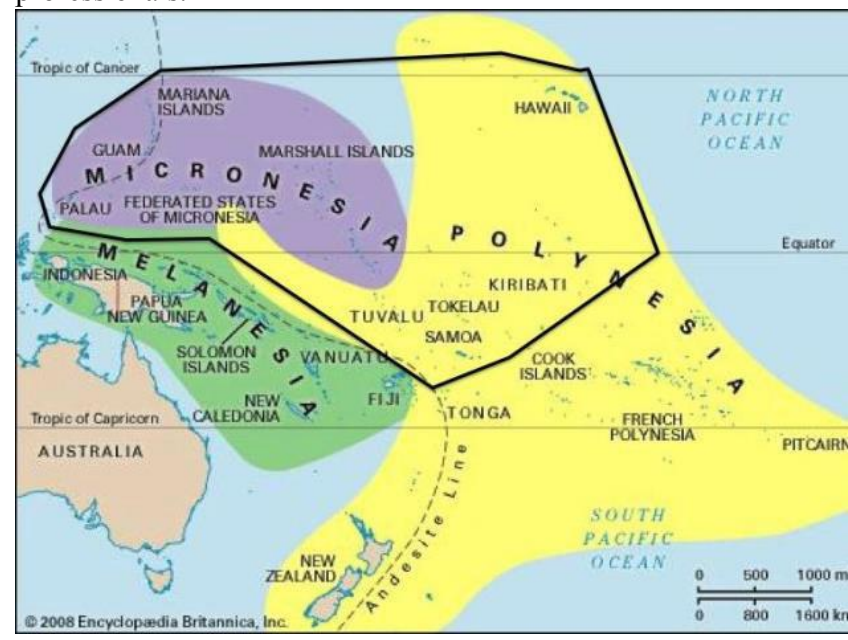

Figure 2: Chaminade's catchment area in Oceania (black outline). The 7 islands of the main Hawaiian group plus $>1000$ islands and atolls of Micronesia and Polynesia [4].

Chaminade University of Honolulu is a Federally-designated Native Hawaiian-Serving and Minority-Serving Institution, with a special mission to serve underrepresented and socioeconomically disadvantaged students from Hawai'i and the US-affiliated Pacific. Chaminade draws students from a $2 \mathrm{M}$ square mile Pacific region (Figure 2) spanning Hawai'ii and, the US-affiliated Pacific (Samoa, Guam, Saipan, the Marshall Islands, and the Federated States of Micronesia --Chuuk, Yap, Pohnpei and Kosrae). The severely challenging social conditions in many of these island locales give the SPICE team a special responsibility to prepare graduates to lead and effect change in their home communities.

\section{SPICE STRATEGIES}

The SPICE strategy has three elements: (1) Perform original research and program development to bridge computation and culture, (2) Deliver a DSAV Summer Immersion Experience (SIE) and (3) Build capacity in data science at a NHPI-serving undergraduate institution. For the purposes of this paper, we limit our discussion to the first two strategies as the third strategy is designed as a long-term vision resulting from the SIE. We include it here for the sake of completeness. Innovative strategy elements in SPICE are summarized as follows:

Culture: SPICE confronts two challenges that contribute to disenfranchisement of NHPI from STEM, and in particular, computationally-intensive careers. SPICE seeks to demonstrate, through original research and the development of new practices, that data science can in fact be culturally-consistent for NHPI. Contextualized by perceptions that the Western STEM paradigm is at best not aligned with the Hawaiian worldview, and at worst represents highly colonized perspectives, SPICE acknowledges that a priori, data science approaches may seem particularly distant from traditional practices and current community needs. Through engaging Native Hawaiian wisdom leaders, community and family in all aspects of the project, SPICE seeks to address alienation and outsider status that are often the lived experience of NHPI who choose STEM careers.

Curriculum: We integrate the collective understanding of the challenge of preparing the next-generation of big data literate scientists, engineers, and innovators to meet workforce demands using a unique recipe: introducing the societal impact of the problem as the motivation and teaching the implementation in that context (teaching the why first, how second), always providing the students with the connection and impact to their lives, families, and communities. The curriculum is being developed as a combination of project-based and socioemotional learning methods that focus on real world challenges, leadership skills, teamwork, and communication skills. We use storytelling [2529], visualization [30-35]; team science [29, 36-38], and discovery-based learning [39-41] to engage the students. Gaming techniques are incorporated to engage, inspire and teach students to create visualizations and virtual environments through freely downloadable gaming engines (e.g. Unity).

Educational Engagement through Socially Relevant Topics: Curricula emphasizing the role of big data for "social good" in informal education settings have demonstrated success at engaging greater proportions of women and underrepresented minorities [42-44]. Infusing social context in STEM teaching allows students to make connections between what they learn in the classroom and what they see in everyday life. Allowing students to make a connection between a subject matter or topic and the world they live in as they continue persisting in their undergraduate program and beyond has been validated as a pedagogical approach because it prepares students to grapple with the interdisciplinary nature of 21 st century problems. Social relevance in curriculum and research projects is foundational to SPICE and uses a flipped model of in-place learning.

\section{SPICE GOALS AND ACTIVIES}




\section{Goal 1. Bridge computation and culture.}

Computationally-intense science is not a priori culturallyconsistent with Pacific indigenous worldviews and traditional practices, and original research is needed to ascertain whether this alignment to culture is possible and how it could be demonstrated in educational activities.

We have assembled a cultural engagement team (CET) that includes a Kumu-in-residence and local faculty members, with the overall goal of developing culturally-consistent conceptual and practical frameworks for thinking about big data and communicating student outcomes and attainment to family, community and kupuna (wisdom figures). Initial activities focus on achieving shared understanding and alignment with pono (Hawaiian for in right relationship) practices within the CET, through immersive orientation and cultural practices.

To perform original scholarly research on cultural consistency and alignment of computationally intensive science, we focus on communicating data science approaches and capabilities in a manner that bridges Western and Hawaiian epistemologies, and developing an example set of the ways in which this type of science contributes to the building of a strong Lāhui (Hawaiian homeland). Products of this work are envisaged as both peerreviewed scholarly work and Ha'i'ólelo (oration/storytelling narratives) for use in community/family outreach and student recruitment. We are investigating a cadre of research questions and storytelling techniques: (1) Can we develop a crosswalk between traditional/historical knowledge, and ways of knowing, and Western approaches to data-intensive science? (2) How is complex knowledge shared, synthesized by master practitioners (e.g., in navigation), and disseminated in large data sets (e.g., exemplified by the kumulipo 2000-line ancient Hawaiian creation chant)? Is there evidence that computationally-intensive science in a Western paradigm can be culturally-consistent with Hawaiian identity and worldview? Our research methodologies are anchored in an ethnographic approach involving a series of iterative collaborations with kupuna to first develop culturally and contextually relevant methods for approaching the specific research questions. Qualitative methods are used to answer these research questions (individual and group interviews, participant observation). Parallel ethnographic assessment of how complex knowledge is shared in Hawaiian and Western settings using interviews, observations of masters and undergraduate students, and review of documents and publications. A bidirectional model informed by ethnographic data informs the similarities/differences of both worldviews/ontologies and epistemologies and is iterated to generate the crosswalk and address the research questions above.

To guide the SIE activities from the viewpoint of oiwi-Pasifika cultural norms and pono practices, the CET in collaboration with the science engagement team are developing culturally-consistent conceptual and practical frameworks for thinking about big data and communicating student outcomes and attainment to family, community and kupuna (wisdom figures). The CET contributes to the implementation of the SIE and building efforts by: (1) offering cultural viewpoints on research projects, specifically focusing on describing to participants their geographical and historical connection to place; (2) designing a suite of pono practices for SPICE participants and their families such as huakai (culturallyrelevant field trips) and a kanikapila-style capstone event in which SPICE participants demonstrate their projects and learning to family and community in a manner normed to Hawaiian practices (e.g., including welcoming hula, demonstrations to kupuna, a shared meal, music); (3) developing gathering protocols for the in silico environment. This effort reflects that in the Hawaiian worldview, access to knowledge and resources has to be earned and one of the vehicles for 'becoming ready' for knowledge is the gathering protocol. Obviously, in silico environments often offer open source knowledge with no personal relationship between the end user and the generator of the information therein. This absence of relationship is antithetical to the Hawaiian practice of learning or investigation. Gathering protocols help bridge this gap, and have been designed previously [48-50] for Western scientists who wish to receive traditional ecological knowledge or enter a sacred place. The digital gathering protocol includes the following elements: (i) reflection on the gathering area and its context in a figurative ahupuaa of resources, (ii) composition (a shared activity with CET and SIE students) of an oli komo (entering chant) which asks permission of the resources' creators and explains the genealogy of the requestor (in this context meaning the path that brought the user to this place and time, not their family genetics), (iii) giving of attribution and credit to the source of the original knowledge, (iv) the leaving of a gift and expression of thanks (e.g., completion of a social justice related task online, contribution to a citizen science project online, leaving of a data product on an open source site).

Products of work are envisaged as both peer-reviewed scholarly work, Ha 'i 'ólelo (oration/storytelling narratives) for use in community/family outreach and student recruitment and curriculum/program materials for SIE. Additionally, the completion of crosswalk and activity design prior to the SIE, and inaugural SIE students and faculty participating in oli komo and digital gathering protocol design are valuable elements of the work as well. New capacity for cultural engagement, new scholarly insights into bridging computation and STEM with indigenous cultures, and improved capacity for dissemination via non-Western outlets allows the SPICE program infused with these culturally-normed practices for Pacific learners, and new digital gathering protocols to be made available to the broader Pacific STEM community.

Goal 2: Deliver a Data Science, Analytics and Visualization (DSAV) Summer Immersion Experience (SIE).

DSAV capacity will enable self-determination and social/economic development in NHPI communities. No relevant training courses or programs are currently available in the Pacific region. We are implementing an in situ summer program in Hawai'i (summer 2019) where we provide a month-long immersion course in data science, visualization, virtual reality and analytics.

To design and refine SIE curriculum with a focus on socially relevant computing, SIE will emphasize project-based learning with themes that are of importance to the Pacific region, require data science approaches and incorporate socially-and culturally- 
relevant research focus areas into the SPICE SIE. The curriculum is being developed as a combination of project-based and socioemotional learning methods that focus on real world challenges and teach students computational skills in the context of those challenges.

We have partnered to identify research areas important to the Pacific region requiring data science approaches to embed placerelevant research and application projects in the SIE. These form the foundation for socially-and culturally-relevant research focus areas to be incorporated into the SPICE SIE. Three proposed project areas are being developed that involve big data, leverage publicly available data sets, are amenable to data science approaches including advanced visualization and virtual reality, and illustrate interfaces between science, traditional culture, and pressing problems in the Pacific region. These include: (i) Network Pharmacology of Pacific indigenous plants that are used for medicinal and ritualistic practice in Hawai' $i$ and the Pacific islands. We can use open source tools such as BATMAN-TCM to deconvolve plant components and predict their effects on human physiology, and reconcile this with cultural understanding derived from kupuna testimony and Hawaiian language sources, and develop new understanding of potential therapeutic approaches derived from indigenous knowledge. (ii) Fisheries science. Use current fisheries data sets to assess future reserves, compare scenarios that involve commercial versus traditional fishing methods, research the impact of National Marine Monument designation for the Papahānaumokuākea area of the Northwestern Hawaiian Islands. (iii) Water sustainability. Of interest in this area are model water availability, test development scenarios and model changes in land use. We can integrate Hawaiian storytelling on water flow and availability over an Arc-GIS framework to provide a culturally-informed water map of these chosen areas. (iv) Visualization of ecological networks present in the Hawaiian language creation chant (kumulipo). Preliminary visualizations of the ecological networks present in the kumulipo offer an opportunity for DSAV to extract traditional understandings of ecological networks and compare them with contemporary understandings from Western science. Research project areas, aggregated data sets and question sets, and projects related to these data sets, with research results disseminated in the form of web reports, web-based visualizations and, where possible, conventional peer-reviewed publications are disseminated.

A new summer short course in DSAV for Pacific participants is being designed, and will be implemented, evaluated, and positioned for expansion to scale in the greater Pacific. Additionally, research and application projects that are culturallyand geographically-aligned with Pacific and community identified and moved forward through DSAV will be disseminated to NHPI families, community, and the greater NHPI population.

\section{SPICE EVALUATION}

We will deliver and evaluate the program using pre/post and follow-up evaluation to assess student learning gains, and socioemotional impacts of the program -specifically targeting 6 assessment towards determining value add of SPICE participation and how this varies across groups (by gender, ethnicity, first year/sophomore status, socioeconomic status, indices of cultural identity, and major), assessing opportunities and barriers to participation in SPICE and outcomes associated with high level engagement (e.g., retention in STEM, computational career goal), and longitudinally, track student GPA at graduation, grade attainment in junior and senior courses with computational content at Chaminade (e.g., Systems Biology, Genomics and Epigenetics) and post-graduate destinations for SPICE participants. To do this, SPICE will employ a robust independent evaluation led by Georgia Tech and designed to provide formative information to guide program improvement and summative assessment of program quality, effectiveness and impact. The evaluation is designed to answer four questions: (1) Implementation: Are SPICE activities being implemented on schedule and as planned? (2) Effectiveness: Are key components of SPICE (CET, MT, SIE, cultural frameworks, Certificate in Data Science) operating effectively and how might they be improved? (3) Impact: What near (participant demographics, participant satisfaction; learning outcomes, socioemotional outcomes) and longer-term outcomes (academic and employment advancement of participants, grant submission, awards, publications, presentations) are associated with participation in SPICE? What is the value-added of the partnership to key stakeholders? (4) Sustainability: How and to what extent are elements of the SPICE scaling and becoming institutionalized? To what extent will aspects of the partnership be expanded and sustained beyond NSF funding? What opportunities and barriers exist?

\section{SUMMARY}

SPICE brings together a diverse team to design, develop and deploy a novel approach to inspire and educate NHPI students to pursue STEM fields and ensure these students gain competency in 'big data'. SPICE recognizes that agency over, and command of, big data is central to NHPI self-determination, and addressing NHPI participation in data science requires new collaborations and partnerships between organizations with geographical separation and different missions. The SPICE pedagogy is anchored with team science and collaborative approaches that blend culture, curriculum and educational materials on NHPI socially-relevant STEM topics. Engaging students with the 'why' before teaching the 'how' and teaching in the context of the Hawaiian culture is a cornerstone to the unique SPICE approach. Using visualization and storytelling as a universal language that crosses cultural, ethnic, gender and discipline-specific boundaries allows the SPICE team to teach students to be successful problem solvers in issues that are both socially important and in need of a workforce literate in computational and data science. The SPICE program uses a flipped model bringing the immersive experience to the students, ensuring that all participants -teachers, students, family, community leaders - gain a sense of team, a sense of place and a respect and appreciation for the NHPI culture. 


\section{ACKNOWLEDGMENTS}

We would like to thank the National Science Foundation for supporting this work, award \#1744526. The project is funded through the National Science Foundation INCLUDES DDLP program.

\section{REFERENCES}

1. Hadfield, M., et al., Recognizing and removing barriers to STEM careers for Native Hawaiians and Pacific Islanders: report on a workshop at the University of Hawaii. 2016.

2. Diamond, J. Connecting Underrepresented Youth to STEM through Culturally Relevant Game Design. Available from: http://ltd.edc.org/connectingunderrepresented-youth-stem-through-culturally-relevant-game-design.

3. Roschelle, J.M., et al., Changing how and what children learn in school with computer-based technologies. Future Child, 2000. 10(2): p. 76-101.

4. Gr, et al., 'Disruptive technologies', 'pedagogical innovation': What's new? Findings from an in-depth study of students' use and perception of technology. Comput. Educ., 2008. 50(2): p. 511-524.

5. Culturally Relevant Teaching.

6. Look, M.A., et al., Kumu hula perspectives on health. Hawaii J Med Public Health, 2014. 73(12 Suppl 3): p. 21-5.

7. Kaholokula, J.K., et al., Sociodemographic, behavioral, and biological variables related to weight loss in native Hawaiians and other Pacific Islanders. Obesity (Silver Spring), 2013. 21(3): p. E196-203.

8. Worth, K., World's Largest Telescope Faces Opposition from Native Hawaiian Protesters, in Scientific American. 2015.

9. Benham, M.K.P., A challenge to Native Hawaiian and Pacific Islander scholars: what the research literature teaches us about our work. Race Ethnicity and Education, 2006. 9(1): p. 29-50.

10. DeBaryshe, B., et al., The roles of family obligation and parenting practices in explaining the well-being of Native Hawaiian adolescents living in poverty. Hulili: Multidisciplinary Research on Hawaiian Well-Being, 2006. 3: p. 103 125 .

11. Tran, J.H., et al., Understanding a Pacific Islander Young Adult Perspective on Access to Higher Education. Calif J Health Promot, 2010. 8: p. 23-38.

12. Alexander, D., Rebuilding the matrix : science and faith in the 21st century. $1 \mathrm{st}$ ed. 2001, Oxford: Lion. 510 p

13. Hezel, F.X., Making Sense of Micronesia: The Logic of Pacific Island Culture. 2013: University of Hawaii Press.

14. McCubbin, L.D., The role of Indigenous family ethnic schema on well-being among Native Hawaiian families. Contemp Nurse, 2006. 23(2): p. 170-80.

15. Native Hawaiian Data Book. 2013.

16. Schools, K., Strategic Planning and Implementation Division. Ka Huaka i. 2014

17. Hokoana, L., Native Hawaiians and College Success: Does Culture Matter?, in Education. 2010, University of Southern California.

18. Ladson-Billings, G., The Dreamkeepers: Successful Teachers of African American Children. 2009: Jossey-Bass.

19. Pukui, M., “Olelo No"'eau: Hawaiian proverbs \& poetical sayings. 1983 : Bishop Museum Press.

20. Curtis, M., The obesity epidemic in the Pacific Islands. Journal of Development and Social Transformation. 1: p. 37-42.

21. Liu, D.M. and C.K. Alameda, Social determinants of health for Native Hawaiian children and adolescents. Hawaii Med J, 2011. 70(11 Suppl 2): p. 9-14.

22. Press, B., Factors Contributing to College Retention in the Native Hawaiian Population. 2003.

23. Cassels, S., Overweight in the Pacific: links between foreign dependence, global food trade, and obesity in the Federated States of Micronesia. Global Health, 2006. 2: p. 10.

24. Pacific, W.H.O.R.O.f.t.W., Diet, food supply and obesity in the Pacific 2003.

25. Keener, V., et al., Climate change and Pacific Islands: Indicators and impacts. Report for the 2012 Pacific Islands Regional Climate Assessment (PIRCA). 2012: Washington, DC: Island Press.

26. Shibuya, E., Security in Oceania: In the 21st Century. 2003, Asia-Pacific Center for Security Studies.

27. Shirey, L., Importance, interest, and selective attention. The role of interest in learning and development. 1992: Lawrence Erlbaum Associates.

28. Hartley, J. and I. Davies, Note-taking: A critical review. Programmed Learning and Educational Technology, 2006. 15(3): p. 207-224.

29. Raz, A. and J. Buhle, Typologies of attentional networks. Nat Rev Neurosci, 2006. 7(5): p. 367-79.

30. Cangelosi, P. and K. Whitt, Teaching through storytelling: an exemplar. International Journal of Nursing Education Scholarship, 2006. 3(1).

31. Caine, R., et al., 12 Brain/Mind Learning Principles in Action: The Fieldbook for Making Connections, Teaching, and the Human Brain. 2005: SAGE Publications.
32. Stenberg, G., Conceptual and perceptual factors in the picture superiority effect. European Journal of Cognitive Psychology, 2007. 18(6): p. 813-847.

33. McBride, D.M. and B. Anne Dosher, A comparison of conscious and automatic memory processes for picture and word stimuli: a process dissociation analysis. Conscious Cogn, 2002. 11(3): p. 423-60.

34. Endestad, T., S. Magnussen, and T. Helstrup, Memory for pictures and words following literal and metaphorical decisions. Imagination, Cognition and Personality, 2003. 23: p. 209-216.

35. Chi, H. and H. Jain, Teaching Computing to STEM Students via Visualization Tools Procedia Computer Science, 2011. 4: p. 1937-1943.

36. Yuan, X., et al., Visualization Tools for Teaching Computer Security. Trans. Comput. Educ., 2010. 9(4): p. 1-28.

37. Bryan, C., et al., Synteny Explorer: An Interactive Visualization Application for Teaching Genome Evolution. IEEE Transactions on Visualization and Computer Graphics, 2017. 23: p. 711-720.

38. Smith, P. and G. Webb, The efficacy of a low-level program visualization tool for teaching programming concepts to novice C programmers. Journal of Educational Computing Research, 2000. 22: p. 187-215.

39. Clark, J. and A. Paivio, Dual coding theory and education. Educational Psychology, 1991. 3: p. 149-210.

40. Disis, M.L. and J.T. Slattery, The road we must take: multidisciplinary team science. Sci Transl Med, 2010. 2(22): p. 22cm9.

41. Post, C., et al., Capitalizing on thought diversity for innovation. IEEE Engineering Management Review, 2011. 1: p. 100-114.

42. (U.S.), N.a.I.T.R.a.D.P., The Federal Big Data Research and Development Strategic Plan. 2016.

43. Meyers, C. and B. Thomas, Promoting Active Learning. Strategies for the College Classroom. 1993: Jossey-Bass Inc.

44. Dunbar, R., The social brain hypothesis. . Evolutionary Anthropology Issues, News, and Reviews, 1998. 6(5): p. 178-190.

45. Silberman, M., Active Learning: 101 Strategies To Teach Any Subject. 1996 Prentice-Hall.

46. Flora, K., J. Bromley, and A. Bracken, Using the Familiar to Teach the Unfamiliar: Active Learning Strategies in Research Methods PsycEXTRA Dataset.

47. National Research Council, D.o.B.a.S.S.a.E., Committee on National Statistics, Panel on Measuring Social and Civic Engagement and Social Cohesion in Surveys, Civic Engagement and Social Cohesion: Measuring Dimensions of Social Capital to Inform Policy. 2014.

48. Namaka, T., A. Whitehead, and H. Fraiola, Traditional gathering of native hula plants in alien-invaded Hawaiian forests: adaptive practices, impacts on alien invasive species and conservation implications. Environ Conserv, 2006. 33: p. 185.

49. Anderson-Fung, P. and K. Maly, Hawaiian Ecosystems and Culture; Why Growing Plants for Lei Helps to Preserve Hawaii's Natural and Cultural Heritage. 2002, University of Hawaii.

50. Native Hawaiian Permission and Release Protocol for ROV Deep Dives in Papahānaumokuākea, Marine National Monument. Office of Hawaiian Affairs. 